\title{
Taxonomy of the order Mononegavirales: second update 2018
}

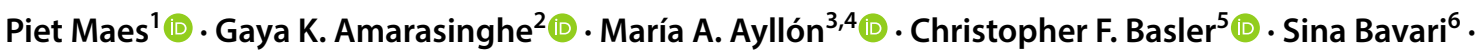

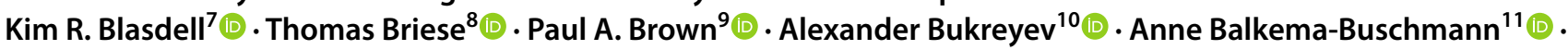

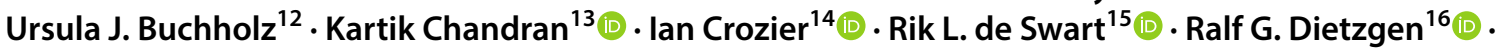

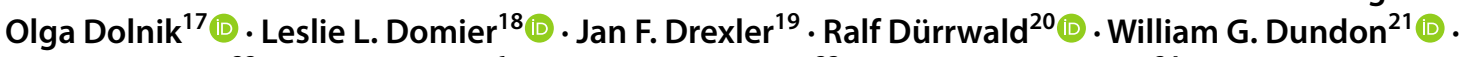 \\ W. Paul Duprex ${ }^{22} \mathbb{D}$. John M. Dye ${ }^{6}$. Andrew J. Easton ${ }^{23} \mathbb{D} \cdot$ Anthony R. Fooks $^{24} \mathbb{D} \cdot$ Pierre B. H. Formenty $^{25} \mathbb{D}$. \\ Ron A. M. Fouchier ${ }^{15}$ (D) Juliana Freitas-Astúa ${ }^{26}$ (D) Elodie Ghedin ${ }^{27}$ (D) Anthony Griffiths ${ }^{28}$ (D) Roger Hewson ${ }^{29}$ (D) . \\ Masayuki Horie $^{30}$ (D) Julia L. Hurwitz ${ }^{31}$ - Timothy H. Hyndman ${ }^{32}$ (D) Dàohóng Jiāng ${ }^{33}$ (D) Gary P. Kobinger ${ }^{34}$ (D) \\ Hideki Kondōo ${ }^{35} \cdot$ Gael Kurath $^{36}$ (D) Ivan V. Kuzmin ${ }^{37}$ (D) $\cdot$ Robert A. Lamb $^{38,39} \cdot$ Benhur Lee $^{40}$ - Eric M. Leroy ${ }^{41}$. \\ Jiànróng Lî ${ }^{42}$. Shin-Yi L. Marzano ${ }^{43}$. Elke Mühlberger ${ }^{28}$ (D) Sergey V. Netesov ${ }^{44}$ (D) Norbert Nowotny ${ }^{45,46}$ (D) \\ Gustavo Palacios $^{6}$ (D) Bernadett Pályi $^{47} \cdot$ Janusz T. Pawęska $^{48}$ (D) Susan L. Payne ${ }^{49}$ (iD $\cdot$ Bertus K. Rima $^{50}$ (iD) \\ Paul Rota ${ }^{51}$ - Dennis Rubbenstroth ${ }^{52}$. Peter Simmonds ${ }^{53}$ (D) Sophie J. Smither ${ }^{54}$. Qisheng Song ${ }^{55}$. \\ Timothy Song ${ }^{27} \cdot$ Kirsten Spann $^{56} \cdot$ Mark D. Stenglein $^{57}$ (D) David M. Stone ${ }^{58}$ (D) Ayato Takada ${ }^{59}$ (D) Robert B. Tesh ${ }^{10}$.

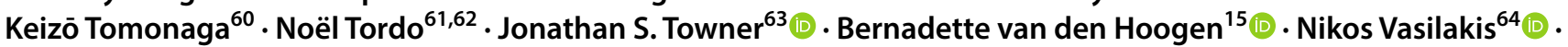 \\ Victoria Wahl ${ }^{65}$ (D) Peter J. Walker ${ }^{66}$ (D) David Wang ${ }^{67,68,69} \cdot$ Lin-Fa Wang $^{70} \cdot$ Anna E. Whitfield $^{71}$ (D)

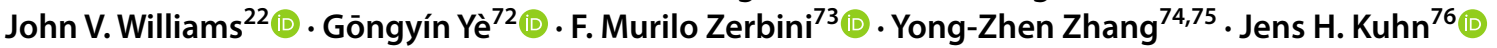

Published online: 20 January 2019

(c) This is a U.S. government work and its text is not subject to copyright protection in the United States; however, its text may be subject to foreign copyright protection 2019

\begin{abstract}
In October 2018, the order Mononegavirales was amended by the establishment of three new families and three new genera, abolishment of two genera, and creation of 28 novel species. This article presents the updated taxonomy of the order Mononegavirales as now accepted by the International Committee on Taxonomy of Viruses (ICTV).
\end{abstract}

Handling Editor: Sead Sabanadzovic.

Thomas Briese, Ralf Dürrwald, Masayuki Horie, Timothy H. Hyndman, Norbert Nowotny, Susan L. Payne, Dennis Rubbenstroth, Mark D. Stenglein, Keizō Tomonaga, Jens H. Kuhn: the members of the 2017-2020 International Committee on Taxonomy of Viruses (ICTV) Bornaviridae Study Group; Gaya K. Amarasinghe, Christopher F. Basler, Sina Bavari, Alexander Bukreyev, Kartik Chandran, Ian Crozier, Olga Dolnik, John M. Dye, Pierre B. H. Formenty, Anthony Griffiths, Roger Hewson, Gary P. Kobinger, Eric M. Leroy, Elke Mühlberger, Sergey V. Netesov, Gustavo Palacios, Bernadett Pályi, Janusz T. Pawęska, Sophie J. Smither, Ayato Takada, Jonathan S. Towner, Victoria Wahl, Jens H. Kuhn: the members of the 2017-2020 ICTV Filoviridae Study Group; Piet Maes, Ralf G. Dietzgen, W. Paul Duprex, Dàohóng Jiāng, Bertus K. Rima, Dennis Rubbenstroth, Peter J. Walker, Yong-Zhen Zhang, Jens H. Kuhn: the members of the 2017-2020 ICTV Mononegavirales Study Group; María A. Ayllón, Dàohóng Jiāng, Shin-Yi L. Marzano: the members of the 2017-2020 ICTV Mymonaviridae Study Group; Ralf G. Dietzgen, Dàohóng Jiāng, Nikos Vasilakis, Jens H. Kuhn: the members of the 2017-2020 ICTV Nyamiviridae Study Group; Anne Balkema-Buschmann, William G. Dundon, W. Paul Duprex, Andrew J. Easton, Ron A. M. Fouchier, Gael Kurath, Robert A. Lamb, Benhur Lee, Bertus K. Rima, Paul Rota, Lin-Fa Wang: the members of the 2017-2020 ICTV Paramyxoviridae Study Group; Paul A. Brown, Rik L. de Swart, Jan F. Drexler, W. Paul Duprex, Andrew J. Easton, Julia L. Hurwitz, Jiànróng Lǐ, Kirsten Spann, Bernadette van den Hoogen, John V. Williams: the members of the 2017-2020 ICTV Pneumoviridae Study Group; Kim R. Blasdell, Ursula J. Buchholz, Ralf G. Dietzgen, Anthony R. Fooks, Juliana Freitas-Astúa, Hideki Kondō, Gael Kurath, Ivan V. Kuzmin, David M. Stone, Robert B. Tesh, Noël Tordo, Nikos Vasilakis, Peter J. Walker, Anna E. Whitfield: the members of the 2017-2020 ICTV Rhabdoviridae Study Group; Peter Simmonds: the 2017-2020 ICTV Chair of the Fungal and Protist Viruses Subcommittee; Peter J. Walker: the 2018-2020 ICTV Proposal Secretary; F. Murilo Zerbini: the 2017-2020 ICTV Chair of the Plant Viruses Subcommittee; and Jens H. Kuhn: the 2017-2020 ICTV Chair of the Animal dsRNA and ssRNA-Viruses Subcommittee.

Extended author information available on the last page of the article 


\section{Introduction}

The virus order Mononegavirales was established in 1991 to accommodate related viruses with nonsegmented, linear, single-stranded, negative-sense RNA genomes classified into three families $[12,13]$. According to the previous ratification vote held in March, 2018, the order included eight families [7]. Amended/emended order descriptions were published in 1995 [4], 1997 [14], 2000 [15], 2005 [16], 2011 [5], 2016 [1], 2017 [2], and March 2018 [3]. Here, we present the changes that were proposed via an official ICTV taxonomic proposal (TaxoProp 2017.016M.A.v1. Mononegavirales_rev) at http://www.ictvonline.org/ in 2017 and were accepted by the ICTV Executive Committee (EC) in October 2018. These changes are now part of the official ICTV taxonomy as of October 2018.

\section{Taxonomic changes at the order rank}

In October 2018, the order Mononegavirales was expanded by three families. The family Artoviridae was created for the established genus Peropuvirus (moved out of Nyamiviridae) and six new species for Běihăi barnacle virus 8 (BhBV-8), Běihăi rhabdo-like virus 1 (BhRLV-1), Běihăi rhabdo-like virus 2 (BhRLV-2), Húběi rhabdo-like virus 5 (HbRLV-5), Húběi rhabdo-like virus 6 (HbRLV-6), and Húběi rhabdo-like virus 8 (HbRLV-8), all discovered in invertebrates $[8,17]$. The family Lispiviridae was established for inclusion of the floating genus Arlivirus; the novel species Hubei arlivirus, Odonate arlivirus, and Wuchang arlivirus for Húběi rhabdo-like virus 3 (HbRLV3), Húběi odonate virus 10 (HbOV-10), and Wǔchāng romanomermis nematode virus $2(\mathrm{WcRNV}-2)$ discovered in invertebrates; and the (renamed) species of the now abandoned free-floating genera Chengtivirus and Wastrivirus $[8,17]$. The family Xinmoviridae was established for the floating genus Anphevirus, and six novel species were added to the genus for Bolahun virus (BLHV), Drosophila unispina virus 1 (DuniV-1), Húběi diptera virus 11 (HbDV-11), Húběi orthoptera virus 5 (HbOV-5), Húběi rhabdo-like virus 7 (HbRLV-7), and Shuāngào fly virus 2 (SgFV-2) discovered in invertebrates $[6,8,9,17]$.

\section{Taxonomic changes at the family rank}

\section{Bornaviridae}

In October 2018, no changes were made at the family rank.

\section{Filoviridae}

In October 2018, no changes were made at the family rank.

\section{Mymonaviridae}

In October 2018, six new species were added to the genus Sclerotimonavirus for Húběi rhabdo-like virus 4 (HbRLV4), Sclerotinia sclerotiorum negative-stranded RNA virus 4 (SsNSRV-4), soybean leaf-associated negative-stranded RNA virus 1 (SLaNSRV-1), soybean leaf-associated negative-stranded RNA virus 2 (SLaNSRV-2), soybean leafassociated negative-stranded RNA virus 3 (SLaNSRV-3), and soybean leaf-associated negative-stranded RNA virus 4 (SLaNSRV-4) associated with soybean leaves, invertebrates, and fungi $[10,11,17]$.

\section{Nyamiviridae}

In October 2018, the free-floating genus Crustavirus was included in the family, and two novel species were added to that genus for Běihăi rhabdo-like virus 6 (BhRLV-6) and Wēnlíng crustacean virus 12 (WlCV-12) discovered in invertebrates. One genus, Peropuvirus, was moved from the family into the new family Artoviridae. Finally, the family was expanded by three novel genera that include a total of five new species for Běihăi rhabdo-like virus 3 (BhRLV-3), Běihăi rhabdo-like virus 4 (BhRLV-4), Běihăi rhabdo-like virus 5 (BhRLV-5), Orinoco virus (ONCV), and Wēnzhōu tapeworm virus 1 (WzTWV-1) discovered in invertebrates $[8,17]$.

\section{Paramyxoviridae}

In October 2018, no changes were made at the family rank.

\section{Pneumoviridae}

In October 2018, no changes were made at the family rank.

\section{Rhabdoviridae}

In October 2018, no changes were made at the family rank.

\section{Sunviridae}

In October 2018, no changes were made at the family rank. 
Table 1 ICTV-accepted taxonomy of the order Mononegavirales as of October 2018. Listed are all mononegaviruses that are classified into species

\begin{tabular}{|c|c|c|}
\hline Genus & Species $^{\text {II }}$ & Virus (Abbreviation) ${ }^{\mathbb{I}}$ \\
\hline \multicolumn{3}{|l|}{ Family Artoviridae } \\
\hline \multirow[t]{7}{*}{ Peropuvirus } & Barnacle peropuvirus & Běihǎi barnacle virus 8 (BhBV-8) \\
\hline & Beihai peropuvirus & Běihǎi rhabdo-like virus 1 (BhRLV-1) \\
\hline & Hubei peropuvirus & Húběi rhabdo-like virus 6 (HbRLV-6) \\
\hline & Odonate peropuvirus & Húběi rhabdo-like virus 8 (HbRLV-8) \\
\hline & Pillworm peropuvirus & Húběi rhabdo-like virus 5 (HbRLV-5) \\
\hline & Pteromalus puparum peropuvirus* & Pteromalus puparum negative-strand RNA virus 1 (PpNSRV-1) \\
\hline & Woodlouse peropuvirus & Běihăi rhabdo-like virus 2 (BhRLV-2) \\
\hline \multicolumn{3}{|c|}{ Family Bornaviridae } \\
\hline \multirow[t]{2}{*}{ Carbovirus } & Queensland carbovirus* & jungle carpet python virus (JCPV) \\
\hline & Southwest carbovirus & southwest carpet python virus (SWCPV) \\
\hline \multirow[t]{16}{*}{ Orthobornavirus } & Elapid 1 orthobornavirus & Loveridge's garter snake virus 1 (LGSV-1) \\
\hline & Mammalian 1 orthobornavirus* & Borna disease virus 1 (BoDV-1) \\
\hline & & Borna disease virus 2 (BoDV-2) \\
\hline & Mammalian 2 orthobornavirus & variegated squirrel bornavirus 1 (VSBV-1) \\
\hline & Passeriform 1 orthobornavirus & canary bornavirus $1(\mathrm{CnBV}-1)$ \\
\hline & & canary bornavirus $2(\mathrm{CnBV}-2)$ \\
\hline & & canary bornavirus 3 (CnBV-3) \\
\hline & Passeriform 2 orthobornavirus & estrildid finch bornavirus 1 (EsBV-1) \\
\hline & Psittaciform 1 orthobornavirus & parrot bornavirus 1 (PaBV-1) \\
\hline & & parrot bornavirus 2 (PaBV-2) \\
\hline & & parrot bornavirus 3 (PaBV-3) \\
\hline & & parrot bornavirus 4 (PaBV-4) \\
\hline & & parrot bornavirus 7 (PaBV-7) \\
\hline & Psittaciform 2 orthobornavirus & parrot bornavirus 5 (PaBV-5) \\
\hline & Waterbird 1 orthobornavirus & aquatic bird bornavirus 1 (ABBV-1) \\
\hline & & aquatic bird bornavirus $2(\mathrm{ABBV}-2)$ \\
\hline \multicolumn{3}{|l|}{ Family Filoviridae } \\
\hline Cuevavirus & Lloviu cuevavirus* & Lloviu virus (LLOV) \\
\hline \multirow[t]{5}{*}{ Ebolavirus } & Bundibugyo ebolavirus & Bundibugyo virus (BDBV) \\
\hline & Reston ebolavirus & Reston virus (RESTV) \\
\hline & Sudan ebolavirus & Sudan virus (SUDV) \\
\hline & Tai Forest ebolavirus & Taï Forest virus (TAFV) \\
\hline & Zaire ebolavirus* & Ebola virus (EBOV) \\
\hline \multirow[t]{2}{*}{ Marburgvirus } & Marburg marburgvirus* & Marburg virus (MARV) \\
\hline & & Ravn virus (RAVV) \\
\hline \multicolumn{3}{|l|}{ Family Lispiviridae } \\
\hline \multirow[t]{6}{*}{ Arlivirus } & Hubei arlivirus & Húběi rhabdo-like virus 3 (HbRLV-3) \\
\hline & Lishi arlivirus* & Líshí spider virus 2 (LsSV-2) \\
\hline & Odonate arlivirus & Húběi odonate virus 10 (HbOV-10) \\
\hline & Gerrid arlivirus & Sānxiá water strider virus 4 (SxWSV-4) \\
\hline & Tacheng arlivirus & Tăchéng tick virus 6 (TcTV-6) \\
\hline & Wuchang arlivirus & Wǔchāng romanomermis nematode virus 2 (WcRNV-2) \\
\hline \multicolumn{3}{|c|}{ Family Mymonaviridae } \\
\hline \multirow[t]{4}{*}{ Sclerotimonavirus } & Dadou sclerotimonavirus & soybean leaf-associated negative-stranded RNA virus 3 (SLaNSRV-3) \\
\hline & Drop sclerotimonavirus & Sclerotinia sclerotiorum negative-stranded RNA virus 2 (SsNSRV-2) \\
\hline & & Sclerotinia sclerotiorum negative-stranded RNA virus 4 (SsNSRV-4) \\
\hline & Glycine sclerotimonavirus & Fusarium graminearum negative-stranded RNA virus 1 (FgNSRV-1) \\
\hline
\end{tabular}


Table 1 (continued)

\begin{tabular}{|c|c|c|}
\hline Genus & Species ${ }^{\mathbb{I}}$ & Virus (Abbreviation) $)^{\mathbb{I}}$ \\
\hline & Hubei sclerotimonavirus & Húběi rhabdo-like virus 4 (HbRLV-4) \\
\hline & Illinois sclerotimonavirus & soybean leaf-associated negative-stranded RNA virus 2 (SLaNSRV-2) \\
\hline & Phyllosphere sclerotimonavirus & soybean leaf-associated negative-stranded RNA virus 4 (SLaNSRV-4) \\
\hline & Sclerotinia sclerotimonavirus $*$ & Sclerotinia sclerotiorum negative-stranded RNA virus 1 (SsNSRV-1) \\
\hline & & Sclerotinia sclerotiorum negative-stranded RNA virus 3 (SsNSRV-3) \\
\hline \multicolumn{3}{|l|}{ Family Nyamiviridae } \\
\hline \multirow[t]{3}{*}{ Berhavirus } & Beihai berhavirus & Běihăi rhabdo-like virus 4 (BhRLV-4) \\
\hline & Echinoderm berhavirus & Běihăi rhabdo-like virus 5 (BhRLV-5) \\
\hline & Sipunculid berhavirus* & Běihăi rhabdo-like virus 3 (BhRLV-3) \\
\hline \multirow[t]{3}{*}{ Crustavirus } & Beihai crustavirus & Běihăi rhabdo-like virus 6 (BhRLV-6) \\
\hline & Wenling crustavirus & Wēnlíng crustacean virus 12 (W1CV-12) \\
\hline & Wenzhou crustavirus* & Wēnzhōu crab virus 1 (WzCV-1) \\
\hline \multirow[t]{3}{*}{ Nyavirus } & Midway nyavirus & Midway virus (MIDWV) \\
\hline & Nyamanini nyavirus* & Nyamanini virus (NYMV) \\
\hline & Sierra Nevada nyavirus & Sierra Nevada virus (SNVV) \\
\hline Orinovirus & Orinoco orinovirus* & Orinoco virus (ONCV) \\
\hline Socyvirus & Soybean cyst nematode socyvirus* & soybean cyst nematode virus 1 (SbCNV-1) \\
\hline Tapwovirus & Tapeworm tapwovirus* & Wēnzhōu tapeworm virus 1 (WzTWV-1) \\
\hline \multicolumn{3}{|c|}{ Family Paramyxoviridae } \\
\hline Aquaparamyxovirus & Salmon aquaparamyxovirus* & Atlantic salmon paramyxovirus (AsaPV) \\
\hline \multirow[t]{19}{*}{ Avulavirus } & Avian avulavirus $1 *$ & avian paramyxovirus $1(\mathrm{APMV}-1)^{1}$ \\
\hline & Avian avulavirus 2 & avian paramyxovirus 2 (APMV-2) \\
\hline & Avian avulavirus 3 & avian paramyxovirus 3 (APMV-3) \\
\hline & Avian avulavirus 4 & avian paramyxovirus 4 (APMV-4) \\
\hline & Avian avulavirus 5 & avian paramyxovirus 5 (APMV-5) \\
\hline & Avian avulavirus 6 & avian paramyxovirus 6 (APMV-6) \\
\hline & Avian avulavirus 7 & avian paramyxovirus 7 (APMV-7) \\
\hline & Avian avulavirus 8 & avian paramyxovirus 8 (APMV-8) \\
\hline & Avian avulavirus 9 & avian paramyxovirus 9 (APMV-9) \\
\hline & Avian avulavirus 10 & avian paramyxovirus 10 (APMV-10) \\
\hline & Avian avulavirus 11 & avian paramyxovirus 11 (APMV-11) \\
\hline & Avian avulavirus 12 & avian paramyxovirus 12 (APMV-12) \\
\hline & Avian avulavirus 13 & avian paramyxovirus 13 (APMV-13) \\
\hline & Avian avulavirus 14 & avian paramyxovirus 14 (APMV-14) \\
\hline & Avian avulavirus 15 & avian paramyxovirus 15 (APMV-15) \\
\hline & Avian avulavirus 16 & avian paramyxovirus 16 (APMV-16) \\
\hline & Avian avulavirus 17 & Antarctic penguin virus A (APV-A) \\
\hline & Avian avulavirus 18 & Antarctic penguin virus B (APV-B) \\
\hline & Avian avulavirus 19 & Antarctic penguin virus $\mathrm{C}$ (APV-C) \\
\hline Ferlavirus & Reptilian ferlavirus* & fer-de-lance virus (FDLV) \\
\hline \multirow[t]{5}{*}{ Henipavirus } & Cedar henipavirus & Cedar virus (CedV) \\
\hline & Ghanaian bat henipavirus & Ghana virus $(\mathrm{GhV})^{2}$ \\
\hline & Hendra henipavirus* & Hendra virus $(\mathrm{HeV})$ \\
\hline & Mojiang henipavirus & Mòjiāng virus (MojV) \\
\hline & Nipah henipavirus & Nipah virus (NiV) \\
\hline \multirow[t]{5}{*}{ Morbillivirus } & Canine morbillivirus & canine distemper virus (CDV) \\
\hline & Cetacean morbillivirus & cetacean morbillivirus (CeMV) \\
\hline & Feline morbillivirus & feline morbillivirus (FeMV) \\
\hline & Measles morbillivirus* & measles virus $(\mathrm{MeV})$ \\
\hline & Small ruminant morbillivirus & peste-des-petits-ruminants virus (PPRV) \\
\hline
\end{tabular}


Table 1 (continued)

\begin{tabular}{|c|c|c|}
\hline Genus & Species ${ }^{\text {II }}$ & Virus (Abbreviation) $^{\mathbb{I}}$ \\
\hline & Phocine morbillivirus & phocine distemper virus (PDV) \\
\hline & Rinderpest morbillivirus & rinderpest virus (RPV) \\
\hline \multirow[t]{5}{*}{ Respirovirus } & Bovine respirovirus 3 & bovine parainfluenza virus 3 (BPIV-3) \\
\hline & Human respirovirus 1 & human parainfluenza virus 1 (HPIV-1) \\
\hline & Human respirovirus 3 & human parainfluenza virus 3 (HPIV-3) \\
\hline & Porcine respirovirus 1 & porcine parainfluenza virus 1 (PPIV-1) \\
\hline & Murine respirovirus* & Sendai virus $(\mathrm{SeV})^{3}$ \\
\hline \multirow[t]{18}{*}{ Rubulavirus } & Achimota rubulavirus 1 & Achimota virus 1 (AchPV-1) \\
\hline & Achimota rubulavirus 2 & Achimota virus 2 (AchPV-2) \\
\hline & Bat mumps rubulavirus & bat mumps virus $(\mathrm{BMV})^{4}$ \\
\hline & Human rubulavirus 2 & human parainfluenza virus 2 (HPIV-2) \\
\hline & Human rubulavirus 4 & human parainfluenza virus 4a (HPIV-4a) \\
\hline & & human parainfluenza virus 4b (HPIV-4b) \\
\hline & Mammalian rubulavirus 5 & parainfluenza virus $5(\mathrm{PIV}-5)^{5}$ \\
\hline & Mapuera rubulavirus & Mapuera virus (MapV) \\
\hline & Menangle rubulavirus & Menangle virus (MenPV) \\
\hline & Mumps rubulavirus* & mumps virus $(\mathrm{MuV})$ \\
\hline & Porcine rubulavirus & La Piedad Michoacán Mexico virus (LPMV) ${ }^{6}$ \\
\hline & Simian rubulavirus & simian virus $41(\mathrm{SV}-41)$ \\
\hline & Sosuga rubulavirus & Sosuga virus (SOSV) \\
\hline & Teviot rubulavirus & Teviot virus (TevPV) \\
\hline & Tioman rubulavirus & Tioman virus (TioPV) \\
\hline & Tuhoko rubulavirus 1 & Tuhoko virus 1 (ThkPV-1) \\
\hline & Tuhoko rubulavirus 2 & Tuhoko virus 2 (ThkPV-2) \\
\hline & Tuhoko rubulavirus 3 & Tuhoko virus 3 (ThkPV-3) \\
\hline \multicolumn{3}{|c|}{ Family Pneumoviridae } \\
\hline \multirow[t]{2}{*}{ Metapneumovirus } & Avian metapneumovirus* & avian metapneumovirus (AMPV) ${ }^{7}$ \\
\hline & Human metapneumovirus & human metapneumovirus (HMPV) \\
\hline \multirow[t]{3}{*}{ Orthopneumovirus } & Bovine orthopneumovirus & bovine respiratory syncytial virus (BRSV) \\
\hline & Human orthopneumovirus* & human respiratory syncytial virus (HRSV) \\
\hline & Murine orthopneumovirus & murine pneumonia virus (MPV) \\
\hline \multicolumn{3}{|c|}{ Family Rhabdoviridae } \\
\hline \multirow[t]{5}{*}{ Almendravirus } & Arboretum almendravirus & Arboretum virus (ABTV) \\
\hline & Balsa almendravirus & Balsa virus (BALV) \\
\hline & Coot Bay almendravirus & Coot Bay virus (CBV) \\
\hline & Puerto Almendras almendravirus* & Puerto Almendras virus (PTAMV) \\
\hline & Rio Chico almendravirus & Rio Chico virus (RCHV) \\
\hline \multirow[t]{4}{*}{ Curiovirus } & Curionopolis curiovirus* & Curionopolis virus (CURV) \\
\hline & Iriri curiovirus & Iriri virus (IRIRV) \\
\hline & Itacaiunas curiovirus & Itacaiunas virus (ITAV) \\
\hline & Rochambeau curiovirus & Rochambeau virus (RBUV) \\
\hline \multirow[t]{7}{*}{ Cytorhabdovirus } & Alfalfa dwarf cytorhabdovirus & alfalfa dwarf virus (ADV) \\
\hline & $\begin{array}{l}\text { Barley yellow striate mosaic cytorhabdovi- } \\
\quad \text { rus }\end{array}$ & barley yellow striate mosaic virus (BYSMV) \\
\hline & Broccoli necrotic yellows cytorhabdovirus & broccoli necrotic yellows virus (BNYV) \\
\hline & $\begin{array}{l}\text { Colocasia bobone disease-associated } \\
\text { cytorhabdovirus }\end{array}$ & Colocasia bobone disease-associated virus $(\mathrm{CBDaV})$ \\
\hline & Festuca leaf streak cytorhabdovirus & Festuca leaf streak virus (FLSV) \\
\hline & Lettuce necrotic yellows cytorhabdovirus* & lettuce necrotic yellows virus (LNYV) \\
\hline & Lettuce yellow mottle cytorhabdovirus & lettuce yellow mottle virus (LYMoV) \\
\hline
\end{tabular}


Table 1 (continued)

\begin{tabular}{|c|c|c|}
\hline Genus & Species ${ }^{\text {II }}$ & Virus (Abbreviation) $^{\mathbb{I}}$ \\
\hline & Northern cereal mosaic cytorhabdovirus & northern cereal mosaic virus (NCMV) \\
\hline & Sonchus cytorhabdovirus 1 & Sonchus virus (SonV) \\
\hline & Strawberry crinkle cytorhabdovirus & strawberry crinkle virus (SCV) \\
\hline & $\begin{array}{l}\text { Wheat American striate mosaic cytorhab- } \\
\text { dovirus }\end{array}$ & wheat American striate mosaic virus (WASMV) \\
\hline \multirow[t]{2}{*}{ Dichorhavirus } & Coffee ringspot dichorhavirus & coffee ringspot virus (CoRSV) \\
\hline & Orchid fleck dichorhavirus* & orchid fleck virus (OFV) \\
\hline \multirow[t]{9}{*}{ Ephemerovirus } & Adelaide River ephemerovirus & Adelaide River virus (ARV) \\
\hline & Berrimah ephemerovirus & Berrimah virus (BRMV) \\
\hline & Bovine fever ephemerovirus* & bovine ephemeral fever virus (BEFV) \\
\hline & Kimberley ephemerovirus & Kimberley virus (KIMV) \\
\hline & & Malakal virus (MALV) \\
\hline & Koolpinyah ephemerovirus & Koolpinyah virus (KOOLV) \\
\hline & Kotonkan ephemerovirus & kotonkan virus (KOTV) \\
\hline & Obodhiang ephemerovirus & Obodhiang virus (OBOV) \\
\hline & Yata ephemerovirus & Yata virus (YATV) \\
\hline \multirow[t]{15}{*}{ Hapavirus } & Flanders hapavirus & Flanders virus (FLAV) \\
\hline & Hart Park hapavirus & Hart Park virus (HPV) \\
\hline & Gray Lodge hapavirus & Gray Lodge virus (GLOV) \\
\hline & Joinjakaka hapavirus & Joinjakaka virus (JOIV) \\
\hline & La Joya hapavirus & La Joya virus (LJV) \\
\hline & Kamese hapavirus & Kamese virus (KAMV) \\
\hline & Landjia hapavirus & Landjia virus (LANV = LJAV) \\
\hline & Manitoba hapavirus & Manitoba virus (MANV = MNTBV) \\
\hline & Marco hapavirus & Marco virus (MCOV) \\
\hline & Mosqueiro hapavirus & Mosqueiro virus (MQOV) \\
\hline & Mossuril hapavirus & Mossuril virus (MOSV) \\
\hline & Ngaingan hapavirus & Ngaingan virus (NGAV) \\
\hline & Ord River hapavirus & Ord River virus (ORV) \\
\hline & Parry Creek hapavirus & Parry Creek virus (PCV) \\
\hline & Wongabel hapavirus* & Wongabel virus (WONV) \\
\hline \multirow[t]{15}{*}{ Ledantevirus } & Barur ledantevirus & Barur virus (BARV) \\
\hline & Fikirini ledantevirus & Fikirini virus (FKRV) \\
\hline & Fukuoka ledantevirus & Fukuoka virus (FUKV) \\
\hline & Kanyawara ledantevirus & Kanyawara virus (KYAV) \\
\hline & Kern Canyon ledantevirus & Kern Canyon virus (KCV) \\
\hline & Keuraliba ledantevirus & Keuraliba virus (KEUV) \\
\hline & Kolente ledantevirus & Kolente virus (KOLEV) \\
\hline & Kumasi ledantevirus & Kumasi rhabdovirus (KRV) \\
\hline & Le Dantec ledantevirus* & Le Dantec virus (LDV) \\
\hline & Mount Elgon bat ledantevirus & Mount Elgon bat virus (MEBV) \\
\hline & Nkolbisson ledantevirus & Nkolbisson virus (NKOV) \\
\hline & Nishimuro ledantevirus & Nishimuro virus (NISV) ${ }^{8}$ \\
\hline & Oita ledantevirus & Oita virus (OITAV) \\
\hline & Wuhan ledantevirus & Wuhan louse fly virus 5 (WLFV-5) \\
\hline & Yongjia ledantevirus & Yongjia tick virus 2 (YTV-2) \\
\hline \multirow[t]{4}{*}{ Lyssavirus } & Aravan lyssavirus & Aravan virus (ARAV) \\
\hline & Australian bat lyssavirus & Australian bat lyssavirus (ABLV) \\
\hline & Bokeloh bat lyssavirus & Bokeloh bat lyssavirus (BBLV) \\
\hline & Duvenhage lyssavirus & Duvenhage virus (DUVV) \\
\hline
\end{tabular}


Table 1 (continued)

\begin{tabular}{|c|c|c|}
\hline Genus & Species ${ }^{\text {II }}$ & Virus (Abbreviation) $^{\mathbb{I}}$ \\
\hline & European bat 1 lyssavirus & European bat lyssavirus 1 (EBLV-1) \\
\hline & European bat 2 lyssavirus & European bat lyssavirus 2 (EBLV-2) \\
\hline & Gannoruwa bat lyssavirus & Gannoruwa bat lyssavirus (GBLV) \\
\hline & Ikoma lyssavirus & Ikoma lyssavirus (IKOV) \\
\hline & Irkut lyssavirus & Irkut virus (IRKV) \\
\hline & Khujand lyssavirus & Khujand virus (KHUV) \\
\hline & Lagos bat lyssavirus & Lagos bat virus (LBV) \\
\hline & Lleida bat lyssavirus & Lleida bat lyssavirus (LLEBV) \\
\hline & Mokola lyssavirus & Mokola virus (MOKV) \\
\hline & Rabies lyssavirus* & rabies virus (RABV) \\
\hline & Shimoni bat lyssavirus & Shimoni bat virus (SHIBV) \\
\hline & West Caucasian bat lyssavirus & West Caucasian bat virus (WCBV) \\
\hline \multirow[t]{4}{*}{ Novirhabdovirus } & Hirame novirhabdovirus & hirame rhabdovirus (HIRV) \\
\hline & Piscine novirhabdovirus & viral hemorrhagic septicemia virus (VHSV) ${ }^{9}$ \\
\hline & Salmonid novirhabdovirus* & infectious hematopoietic necrosis virus (IHNV) \\
\hline & Snakehead novirhabdovirus & snakehead rhabdovirus (SHRV) \\
\hline \multirow[t]{11}{*}{ Nucleorhabdovirus } & Datura yellow vein nucleorhabdovirus & datura yellow vein virus (DYVV) \\
\hline & Eggplant mottled dwarf nucleorhabdovirus & eggplant mottled dwarf virus (EMDV) \\
\hline & Maize fine streak nucleorhabdovirus & maize fine streak virus (MSFV) \\
\hline & Maize Iranian mosaic nucleorhabdovirus & maize Iranian mosaic virus (MIMV) \\
\hline & Maize mosaic nucleorhabdovirus & maize mosaic virus (MMV) \\
\hline & Potato yellow dwarf nucleorhabdovirus* & potato yellow dwarf virus (PYDV) \\
\hline & Rice yellow stunt nucleorhabdovirus & rice yellow stunt virus (RYSV) \\
\hline & & rice transitory yellowing virus (RTYV) \\
\hline & Sonchus yellow net nucleorhabdovirus & Sonchus yellow net virus (SYNV) \\
\hline & Sowthistle yellow vein nucleorhabdovirus & sowthistle yellow vein virus (SYVV) \\
\hline & Taro vein chlorosis nucleorhabdovirus & taro vein chlorosis virus (TaVCV) \\
\hline \multirow[t]{3}{*}{ Perhabdovirus } & Anguillid perhabdovirus & eel virus European X (EVEX) \\
\hline & Perch perhabdovirus* & perch rhabdovirus (PRV) \\
\hline & Sea trout perhabdovirus & lake trout rhabdovirus (LTRV) \\
\hline \multirow[t]{7}{*}{ Sigmavirus } & Drosophila affinis sigmavirus & Drosophila affinis sigmavirus (DAffSV) \\
\hline & Drosophila ananassae sigmavirus & Drosophila ananassae sigmavirus (DAnaSV) \\
\hline & Drosophila immigrans sigmavirus & Drosophila immigrans sigmavirus (DImmSV) \\
\hline & Drosophila melanogaster sigmavirus* & Drosophila melanogaster sigmavirus (DMelSV) \\
\hline & Drosophila obscura sigmavirus & Drosophila obscura sigmavirus (DObsSV) \\
\hline & Drosophila tristis sigmavirus & Drosophila tristis sigmavirus (DTriSV) \\
\hline & Muscina stabulans sigmavirus & Muscina stabulans sigmavirus (MStaSV) \\
\hline \multirow[t]{4}{*}{ Sprivivirus } & Carp sprivivirus* & spring viremia of carp virus (SVCV) \\
\hline & Pike fry sprivivirus & grass carp rhabdovirus (GrCRV) \\
\hline & & pike fry rhabdovirus (PFRV) \\
\hline & & tench rhabdovirus (TenRV) \\
\hline \multirow[t]{5}{*}{ Sripuvirus } & Almpiwar sripuvirus & Almpiwar virus (ALMV) \\
\hline & Chaco sripuvirus & Chaco virus (CHOV) \\
\hline & Niakha sripuvirus* & Niakha virus (NIAV) \\
\hline & Sena Madureira sripuvirus & Sena Madureira virus (SMV) \\
\hline & Sripur sripuvirus & Sripur virus (SRIV) \\
\hline \multirow[t]{4}{*}{ Tibrovirus } & Bas Congo tibrovirus & Bas-Congo virus (BASV) \\
\hline & Beatrice Hill tibrovirus & Beatrice Hill virus (BHV) \\
\hline & Coastal Plains tibrovirus & Coastal Plains virus (CPV) \\
\hline & Ekpoma 1 tibrovirus & Ekpoma virus 1 (EKV-1) \\
\hline
\end{tabular}


Table 1 (continued)

\begin{tabular}{|c|c|c|}
\hline Genus & Species $^{\text {II }}$ & Virus (Abbreviation) ${ }^{\mathbb{I}}$ \\
\hline & Ekpoma 2 tibrovirus & Ekpoma virus 2 (EKV-2) \\
\hline & Sweetwater Branch tibrovirus & Sweetwater Branch virus (SWBV) \\
\hline & Tibrogargan tibrovirus* & Bivens Arm virus (BAV) \\
\hline & & Tibrogargan virus (TIBV) \\
\hline \multirow[t]{3}{*}{ Tupavirus } & Durham tupavirus* & Durham virus (DURV) \\
\hline & Klamath tupavirus & Klamath virus (KLAV) \\
\hline & Tupaia tupavirus & tupaia virus (TUPV) \\
\hline Varicosavirus & Lettuce big-vein associated varicosavirus* & lettuce big-vein associated virus $(\mathrm{LBVaV})^{10}$ \\
\hline \multirow[t]{16}{*}{ Vesiculovirus } & Alagoas vesiculovirus & vesicular stomatitis Alagoas virus (VSAV) \\
\hline & American bat vesiculovirus & American bat vesiculovirus (ABVV) \\
\hline & Carajas vesiculovirus & Carajas virus (CJSV) \\
\hline & Chandipura vesiculovirus & Chandipura virus (CHPV) \\
\hline & Cocal vesiculovirus & Cocal virus $(\mathrm{COCV})$ \\
\hline & Indiana vesiculovirus* & vesicular stomatitis Indiana virus (VSIV) \\
\hline & Isfahan vesiculovirus & Isfahan virus (ISFV) \\
\hline & Jurona vesiculovirus & Jurona virus (JURV) \\
\hline & Malpais Spring vesiculovirus & Malpais Spring virus (MSPV) \\
\hline & Maraba vesiculovirus & Maraba virus (MARAV) \\
\hline & Morreton vesiculovirus & Morreton virus (MORV) \\
\hline & New Jersey vesiculovirus & vesicular stomatitis New Jersey virus (VSNJV) \\
\hline & Perinet vesiculovirus & Perinet virus (PERV) \\
\hline & Piry vesiculovirus & Piry virus (PIRYV) \\
\hline & Radi vesiculovirus & Radi virus (RADV) \\
\hline & Yug Bogdanovac vesiculovirus & Yug Bogdanovac virus (YBV) \\
\hline Unassigned & Moussa virus & Moussa virus (MOUV) \\
\hline \multicolumn{3}{|c|}{ Family Sunviridae } \\
\hline Sunshinevirus & Reptile sunshinevirus $1^{*}$ & Sunshine Coast virus (SunCV) \\
\hline \multicolumn{3}{|c|}{ Family Xinmoviridae } \\
\hline \multirow[t]{8}{*}{ Anphevirus } & Bolahun anphevirus & Bolahun virus (BLHV) \\
\hline & & Gambie virus (GAMV) \\
\hline & Dipteran anphevirus & Húběi diptera virus 11 (HbDV-11) \\
\hline & Drosophilid anphevirus & Drosophila unispina virus 1 (DuniV-1) \\
\hline & Odonate anphevirus & Húběi rhabdo-like virus 7 (HbRLV-7) \\
\hline & Orthopteran anphevirus & Húběi orthoptera virus 5 (HbOV-5) \\
\hline & Shuangao anphevirus & Shuāngào fly virus 2 (SgFV-2) \\
\hline & Xincheng anphevirus* & Xīnchéng mosquito virus (XcMV) \\
\hline
\end{tabular}

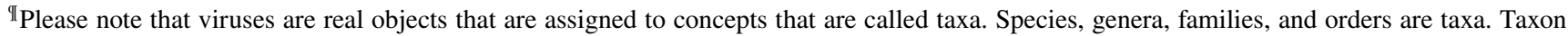
names are always italicized and always begin with a capital letter. Virus names, on the other hand, are not italicized and are not capitalized, except if the name or a name component is a proper noun. This column lists the virus names with their correct (lack of) capitalization. Lists of viruses within a given species are provisional at this point and will likely be amended in the near future
} 


\section{Summary}

A summary of the current, ICTV-accepted taxonomy of the order Mononegavirales is presented in Table 1.

Acknowledgements We thank Laura Bollinger (NIH/NIAID Integrated Research Facility at Fort Detrick, Frederick, MD, USA) for critically editing the manuscript.

Funding This work was supported in part through Battelle Memorial Institute's prime contract with the US National Institute of Allergy and Infectious Diseases (NIAID) under Contract no. HHSN272200700016I (J.H.K.). This project has been funded in whole or in part with federal funds from the National Cancer Institute, National Institutes of Health, under Contract No. HHSN261200800001E (I.C.). This work was also funded in part under Contract No. HSHQDC-15-C-00064 awarded by DHS S\&T for the management and operation of the National Biodefense Analysis and Countermeasures Center (NBACC), a federally funded research and development center (V.W.); and National Institutes of Health (NIH) contract HHSN272201000040I/HHSN27200004/ D04 and Grant R24AI120942 (N.V., R.B.T.). This work was also supported by the UK Department for Environment, Food and Rural Affairs (Defra), Scottish Government and Welsh Government (Grant number SV3500) (A.R.F.).

\section{Compliance with ethical standards}

The views and conclusions contained in this document are those of the authors and should not be interpreted as necessarily representing the official policies, either expressed or implied, of the US Department of the Army, the US Department of Defense, the US Department of Health and Human Services, the Department of Homeland Security (DHS) Science and Technology Directorate (S\&T), or of the institutions and companies affiliated with the authors. In no event shall any of these entities have any responsibility or liability for any use, misuse, inability to use, or reliance upon the information contained herein. The US departments do not endorse any products or commercial services mentioned in this publication.

Conflict of interest The authors declare no conflicts of interest.

Ethical approval This article does not contain any studies with human participants or animals performed by any of the authors.

\section{References}

1. Afonso CL, Amarasinghe GK, Bányai K, Bào Y, Basler CF, Bavari S, Bejerman N, Blasdell KR, Briand F-X, Briese T, Bukreyev A, Calisher CH, Chandran K, Chéng J, Clawson AN, Collins PL, Dietzgen RG, Dolnik O, Domier LL, Dürrwald R, Dye JM, Easton AJ, Ebihara H, Farkas SL, Freitas-Astúa J, Formenty P, Fouchier RA, Fù Y, Ghedin E, Goodin MM, Hewson R, Horie M, Hyndman TH, Jiāng D, Kitajima EW, Kobinger GP, Kondo H, Kurath G, Lamb RA, Lenardon S, Leroy EM, Li C-X, Lin X-D, Liú L, Longdon B, Marton S, Maisner A, Mühlberger E, Netesov SV, Nowotny N, Patterson JL, Payne SL, Paweska JT, Randall RE, Rima BK, Rota P, Rubbenstroth D, Schwemmle M, Shi M, Smither SJ, Stenglein MD, Stone DM, Takada A, Terregino C, Tesh RB, Tian J-H, Tomonaga K, Tordo N, Towner JS, Vasilakis N, Verbeek M, Volchkov VE, Wahl-Jensen V, Walsh JA, Walker
PJ, Wang D, Wang L-F, Wetzel T, Whitfield AE, Xiè JT, Yuen K-Y, Zhang Y-Z, Kuhn JH (2016) Taxonomy of the order Mononegavirales: update 2016. Arch Virol 161:2351-2360

2. Amarasinghe GK, Bào Y, Basler CF, Bavari S, Beer M, Bejerman N, Blasdell KR, Bochnowski A, Briese T, Bukreyev A, Calisher $\mathrm{CH}$, Chandran K, Collins PL, Dietzgen RG, Dolnik O, Dürrwald R, Dye JM, Easton AJ, Ebihara H, Fang Q, Formenty P, Fouchier RAM, Ghedin E, Harding RM, Hewson R, Higgins CM, Hong J, Horie M, James AP, Jiāng D, Kobinger GP, Kondo H, Kurath G, Lamb RA, Lee B, Leroy EM, Li M, Maisner A, Mühlberger E, Netesov SV, Nowotny N, Patterson JL, Payne SL, Paweska JT, Pearson MN, Randall RE, Revill PA, Rima BK, Rota P, Rubbenstroth D, Schwemmle M, Smither SJ, Song Q, Stone DM, Takada A, Terregino C, Tesh RB, Tomonaga K, Tordo N, Towner JS, Vasilakis N, Volchkov VE, Wahl-Jensen V, Walker PJ, Wang B, Wang D, Wang F, Wang L-F, Werren JH, Whitfield AE, Yan Z, Ye G, Kuhn JH (2017) Taxonomy of the order Mononegavirales: update 2017. Arch Virol 162:2493-2504

3. Amarasinghe GK, Ceballos NGA, Banyard AC, Basler CF, Bavari S, Bennett AJ, Blasdell KR, Briese T, Bukreyev A, Caì Y, Calisher $\mathrm{CH}$, Lawson CC, Chandran K, Chapman CA, Chiu CY, Choi K-S, Collins PL, Dietzgen RG, Dolja VV, Dolnik O, Domier LL, Dürrwald R, Dye JM, Easton AJ, Ebihara H, Echevarría JE, Fooks AR, Formenty PBH, Fouchier RAM, Freuling CM, Ghedin E, Goldberg TL, Hewson R, Horie M, Hyndman TH, Jiāng D, Kityo R, Kobinger GP, Kondō H, Koonin EV, Krupovic M, Kurath G, Lamb RA, Lee B, Leroy EM, Maes P, Maisner A, Marston DA, Mor SK, Müller T, Mühlberger E, Ramírez VMN, Netesov SV, Ng TFF, Nowotny N, Palacios G, Patterson JL, Pawęska JT, Payne SL, Prieto K, Rima BK, Rota P, Rubbenstroth D, Schwemmle M, Siddell S, Smither SJ, Song Q, Song T, Stenglein MD, Stone DM, Takada A, Tesh RB, Thomazelli LM, Tomonaga K, Tordo N, Towner JS, Vasilakis N, Vázquez-Morón S, Verdugo C, Volchkov VE, Wahl V, Walker PJ, Wang D, Wang L-F, Wellehan JFX, Wiley MR, Whitfield AE, Wolf YI, Yè G, Zhāng Y-Z, Kuhn JH (2018) Taxonomy of the order Mononegavirales: update 2018. Arch Virol 163:2283-2294

4. Bishop DHL, Pringle CR (1995) Order Mononegavirales. In: Murphy FA, Fauquet CM, Bishop DHL, Ghabrial SA, Jarvis AW, Martelli GP, Mayo MA, Summers MD (eds) Virus taxonomy-sixth report of the International Committee on Taxonomy of Viruses/Archives of virology supplement 10. Springer, Vienna, pp 265-267

5. Easton AJ, Pringle CR (2011) Order Mononegavirales. In: King AMQ, Adams MJ, Carstens EB, Lefkowitz EJ (eds) Virus taxonomy-ninth report of the International Committee on Taxonomy of Viruses. Elsevier/Academic Press, London, pp 653-657

6. Fauver JR, Grubaugh ND, Krajacich BJ, Weger-Lucarelli J, Lakin SM, Fakoli LS III, Bolay FK, Diclaro JW II, Dabiré KR, Foy BD, Brackney DE, Ebel GD, Stenglein MD (2016) West African Anopheles gambiae mosquitoes harbor a taxonomically diverse virome including new insect-specific flaviviruses, mononegaviruses, and totiviruses. Virology 498:288-299

7. King AMQ, Lefkowitz EJ, Mushegian AR, Adams MJ, Dutilh BE, Gorbalenya AE, Harrach B, Harrison RL, Junglen S, Knowles NJ, Kropinski AM, Krupovic M, Kuhn JH, Nibert ML, Rubino L, Sabanadzovic S, Sanfaçon H, Siddell SG, Simmonds P, Varsani A, Zerbini FM, Davison AJ (2018) Changes to taxonomy and the International Code of Virus Classification and Nomenclature ratified by the International Committee on Taxonomy of Viruses (2018). Arch Virol 163:2601-2631

8. Li C-X, Shi M, Tian J-H, Lin X-D, Kang Y-J, Chen L-J, Qin X-C, $\mathrm{Xu}$ J, Holmes EC, Zhang Y-Z (2015) Unprecedented genomic diversity of RNA viruses in arthropods reveals the ancestry of negative-sense RNA viruses. Elife 4:e05378 
9. Longdon B, Murray GG, Palmer WJ, Day JP, Parker DJ, Welch JJ, Obbard DJ, Jiggins FM (2015) The evolution, diversity, and host associations of rhabdoviruses. Virus Evol 1:vev014

10. Marzano S-YL, Domier LL (2016) Novel mycoviruses discovered from metatranscriptomics survey of soybean phyllosphere phytobiomes. Virus Res 213:332-342

11. Marzano S-YL, Nelson BD, Ajayi-Oyetunde O, Bradley CA, Hughes TJ, Hartman GL, Eastburn DM, Domier LL (2016) Identification of diverse mycoviruses through metatranscriptomics characterization of the viromes of five major fungal plant pathogens. J Virol 90:6846-6863

12. Pringle CR (1991) Order Mononegavirales. In: Francki RIB, Fauquet CM, Knudson DL, Brown F (eds) Classification and nomenclature of viruses-fifth report of the International Committee on Taxonomy of Viruses/Archives of virology supplementum 2. Springer, Vienna, pp 239-241

13. Pringle CR, Alexander DJ, Billeter MA, Collins PL, Kingsbury DW, Lipkind MA, Nagai Y, Orvell C, Rima B, Rott R, ter Meulen V (1991) The order Mononegavirales. Arch Virol 117:137-140
14. Pringle CR (1997) The order Mononegavirales — current status. Arch Virol 142:2321-2326

15. Pringle CR (2000) Order Mononegavirales. In: van Regenmortel MHV, Fauquet CM, Bishop DHL, Carstens EB, Estes MK, Lemon SM, Maniloff J, Mayo MA, McGeoch DJ, Pringle CR, Wickner RB (eds) Virus taxonomy - seventh report of the International Committee on Taxonomy of Viruses. Academic Press, San Diego, pp 525-530

16. Pringle CR (2005) Order Mononegavirales. In: Fauquet CM, Mayo MA, Maniloff J, Desselberger U, Ball LA (eds) Virus taxonomy - eighth report of the International Committee on Taxonomy of Viruses. Elsevier/Academic Press, San Diego, pp 609-614

17. Shi M, Lin X-D, Tian J-H, Chen L-J, Chen X, Li C-X, Qin X-C, Li J, Cao J-P, Eden J-S, Buchmann J, Wang W, Xu J, Holmes EC, Zhang Y-Z (2016) Redefining the invertebrate RNA virosphere. Nature 540:539-543

\section{Affiliations}

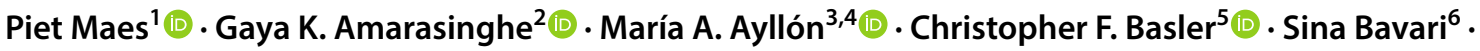

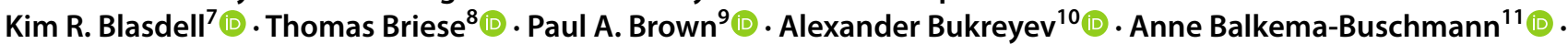

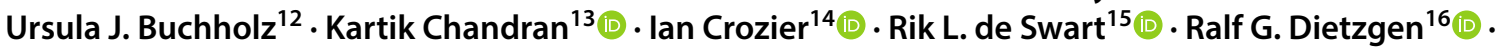

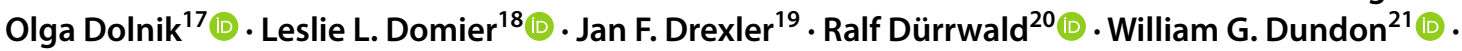

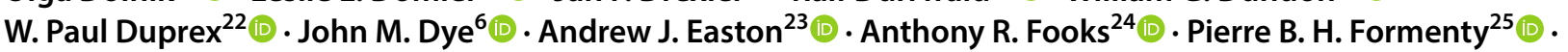

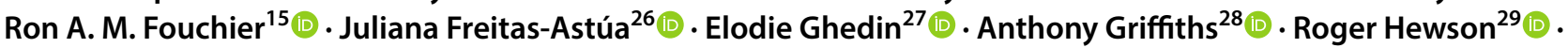

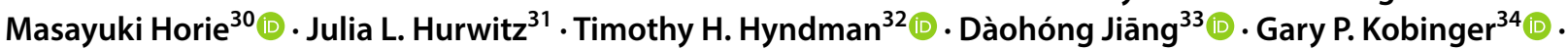
Hideki Kondō ${ }^{35}$. Gael Kurath ${ }^{36}$ - Ivan V. Kuzmin ${ }^{37}$ (1) Robert A. Lamb ${ }^{38,39} \cdot$ Benhur Lee $^{40}$ (1) Eric M. Leroy ${ }^{41}$.

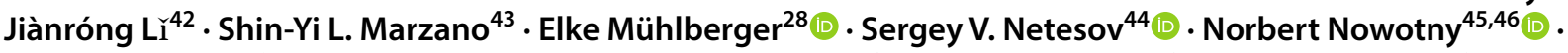

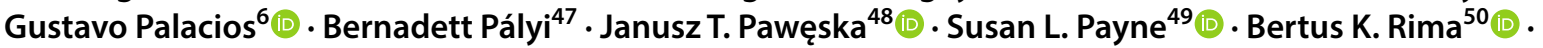
Paul Rota ${ }^{51}$. Dennis Rubbenstroth ${ }^{52} \cdot$ Peter Simmonds $^{53}$ (1) Sophie J. Smither ${ }^{54}$. Qisheng Song ${ }^{55}$. Timothy Song ${ }^{27} \cdot$ Kirsten Spann ${ }^{56} \cdot$ Mark D. Stenglein $^{57}$ - David M. Stone ${ }^{58}$ (1) - Ayato Takada ${ }^{59}$ - Robert B. Tesh ${ }^{10}$.

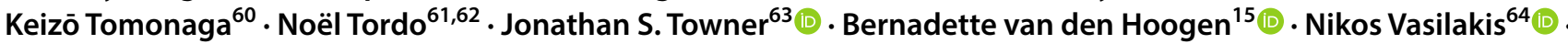
Victoria Wahl ${ }^{65}$ (D) Peter J. Walker ${ }^{66}$ (D) . David Wang ${ }^{67,68,69} \cdot$ Lin-Fa Wang $^{70} \cdot$ Anna E. Whitfield $^{71}$ (1) .

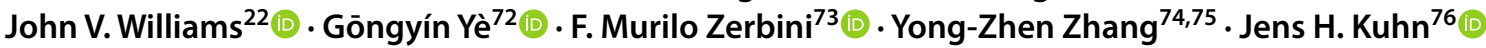

Jens H. Kuhn

kuhnjens@mail.nih.gov

1 Zoonotic Infectious Diseases Unit, Rega Institute, KU Leuven, Leuven, Belgium

2 Department of Pathology and Immunology, Washington University School of Medicine, St. Louis, MO, USA

3 Universidad Politécnica de Madrid-Instituto Nacional de Investigación y Tecnología Agraria y Alimentaria, Campus de Montegancedo, Pozuelo de Alarcón, Madrid, Spain

4 Departamento de Biotecnología-Biología Vegetal, Escuela Técnica Superior de Ingeniería Agronómica, Alimentaria y de Biosistemas, UPM, Madrid, Spain

5 Center for Microbial Pathogenesis, Institute for Biomedical Sciences, Georgia State University, Atlanta, GA, USA

6 United States Army Medical Research Institute of Infectious Diseases, Fort Detrick, Frederick, MD, USA
7 CSIRO Health and Biosecurity, Australian Animal Health Laboratory, Geelong, VIC, Australia

8 Department of Epidemiology, Center for Infection and Immunity, Mailman School of Public Health, Columbia University, New York, NY, USA

9 VIPAC Unit, Agence Nationale de Sécurité Sanitaire, Laboratoire de Ploufragan-Plouzané, Université Bretagne Loire, Ploufragan, France

10 Department of Pathology, The University of Texas Medical Branch, Galveston, TX 77555, USA

11 Friedrich-Loeffler-Institut, Federal Research Institute for Animal Health, Institute of Novel and Emerging Infectious Diseases, Greifswald-Insel Riems, Germany

12 RNA Viruses Section, Laboratory of Infectious Diseases, National Institute of Allergy and Infectious Diseases, National Institutes of Health, Bethesda, MD, USA 
13 Department of Microbiology and Immunology, Albert Einstein College of Medicine, Bronx, NY, USA

14 Integrated Research Facility at Fort Detrick (IRF-Frederick), Clinical Monitoring Research Program Directorate, Frederick National Laboratory for Cancer Research sponsored by the National Cancer Institute, Frederick, MD, USA

15 Department of Viroscience, Erasmus MC, University Medical Centre Rotterdam, Rotterdam, the Netherlands

16 Queensland Alliance for Agriculture and Food Innovation, The University of Queensland, St. Lucia, QLD, Australia

17 Institute of Virology, Philipps University Marburg, Marburg, Germany

18 Department of Crop Sciences, University of Illinois, Urbana, IL, USA

19 Charité-Universitätsmedizin Berlin, corporate member of Freie Universität Berlin, Humboldt-Universität zu Berlin, and Berlin Institute of Health, Institute of Virology, Berlin, Germany Robert Koch Institut, Berlin, Germany

21 Animal Production and Health Laboratory, Joint FAO/IAEA Division of Nuclear Techniques in Food and Agriculture, Department of Nuclear Sciences and Applications, International Atomic Energy Agency, Vienna, Austria

22 University of Pittsburgh School of Medicine, Pittsburgh, PA, USA

23 School of Life Sciences, University of Warwick, Coventry, UK

24 Animal and Plant Health Agency, Weybridge, Surrey, UK

25 World Health Organization, Geneva, Switzerland

26 Embrapa Cassava and Fruits, Cruz das Almas, Bahia, Brazil

27 Department of Biology, Center for Genomics and Systems Biology, New York University, New York, NY, USA

28 Department of Microbiology and National Emerging Infectious Diseases Laboratories, Boston University School of Medicine, Boston, MA, USA

29 Public Health England, Porton Down, Salisbury, Wiltshire, UK

30 Hakubi Center for Advanced Research, Kyoto University, Kyoto, Japan

31 Department of Infectious Diseases, St. Jude Children's Research Hospital, Memphis, TN, USA

32 College of Veterinary Medicine, School of Veterinary and Life Sciences, Murdoch University, Murdoch, WA, Australia

33 State Key Laboratory of Agricultural Microbiology, College of Plant Science and Technology, The Provincial Key Lab of Plant Pathology of Húběi Province, Huázhōng Agricultural University, Wuhan, China

34 Department of Microbiology, Immunology and Infectious Diseases, Université Laval, Quebec City, Canada

35 Institute of Plant Science and Resources, Okayama University, Kurashiki, Japan

36 US Geological Survey Western Fisheries Research Center, Seattle, WA, USA
37 US Department of Agriculture, Animal and Plant Health Inspection, National Veterinary Services Laboratories, Diagnostic Virology Laboratory, Ames, IA, USA

38 Department of Molecular Biosciences, Northwestern University, Evanston, IL, USA

39 Howard Hughes Medical Institute, Northwestern University, Evanston, IL, USA

40 Department of Microbiology, Icahn School of Medicine at Mount Sinai, New York, NY, USA

41 Centre International de Recherches Médicales de Franceville, Institut de Recherche pour le Développement, Franceville, Gabon

42 Department of Veterinary Biosciences, College of Veterinary Medicine, The Ohio State University, Columbus, OH, USA

43 Department of Biology and Microbiology, Department of Plant Sciences, South Dakota State University, Brookings, SD, USA

44 Novosibirsk State University, Novosibirsk, Novosibirsk Oblast, Russia

45 Institute of Virology, University of Veterinary Medicine, Vienna, Austria

46 Department of Basic Medical Sciences, College of Medicine, Mohammed Bin Rashid University of Medicine and Health Sciences, Dubai, United Arab Emirates

47 National Biosafety Laboratory, National Public Health Center, Budapest, Hungary

48 Center for Emerging Zoonotic and Parasitic Diseases, National Institute for Communicable Diseases of the National Health Laboratory Service, Sandringham-Johannesburg, Gauteng, South Africa

49 Department of Veterinary Pathobiology, College of Veterinary Medicine and Biomedical Sciences, Texas A\&M University, College Station, TX, USA

50 Centre for Experimental Medicine, School of Medicine, Dentistry and Biomedical Sciences, The Queen's University of Belfast, Belfast, Northern Ireland, UK

51 National Center for Immunization and Respiratory Diseases, Centers for Disease Control and Prevention, Atlanta, GA, USA

52 Institute of Diagnostic Virology, Friedrich-Loeffler-Institut, Greifswald-Insel Riems, Germany

53 Nuffield Department of Medicine, University of Oxford, Oxford, UK

54 CBR Division, Dstl, Porton Down, Salisbury, Wiltshire, UK

55 Division of Plant Sciences, College of Agriculture, Food and Natural Resources, University of Missouri, Columbia, MO, USA

56 Australian Infectious Diseases Research Centre, University of Queensland, St. Lucia, QLD, Australia

57 Department of Microbiology, Immunology, and Pathology, College of Veterinary Medicine and Biomedical Sciences, Colorado State University, Fort Collins, CO, USA

58 Centre for Environment, Fisheries and Aquaculture Science, Weymouth, Dorset, UK 
59 Division of Global Epidemiology, Hokkaido University Research Center for Zoonosis Control, Sapporo, Japan

60 Institute for Frontier Life and Medical Sciences (inFront), Kyoto University, Kyoto, Japan

61 Institut Pasteur, Unité des Stratégies Antivirales, WHO Collaborative Centre for Viral Haemorrhagic Fevers and Arboviruses, OIE Reference Laboratory for RVFV and CCHFV, Paris, France

62 Institut Pasteur de Guinée, Conakry, Guinea

63 Viral Special Pathogens Branch, Division of High-Consequence Pathogens Pathology, National Center for Emerging and Zoonotic Infectious Diseases, Centers for Disease Control and Prevention, Atlanta, GA, USA

64 Department of Pathology and Center for Biodefense and Emerging Infectious Diseases, The University of Texas Medical Branch, Galveston, TX 77555, USA

65 National Biodefense Analysis and Countermeasures Center, Fort Detrick, Frederick, MD, USA

66 School of Biological Sciences, University of Queensland, St. Lucia, QLD, Australia

67 Department of Molecular Microbiology, Washington University School of Medicine, St. Louis, MO, USA

68 Department of Pathology, Washington University School of Medicine, St. Louis, MO, USA
69 Department of Immunology, Washington University School of Medicine, St. Louis, MO, USA

70 Programme in Emerging Infectious Diseases, Duke-NUS Medical School, Singapore, Singapore

71 Department of Entomology and Plant Pathology, North Carolina State University, Raleigh, NC, USA

72 State Key Laboratory of Rice Biology and Ministry of Agriculture Key Lab of Molecular Biology of Crop Pathogens and Insects, Institute of Insect Sciences, Zhejiang University, Hangzhou, China

73 Departamento de Fitopatologia, Instituto de Biotecnologia Aplicada à Agropecuária, Universidade Federal de Viçosa, Viçosa, MG, Brazil

74 National Institute for Communicable Disease Control and Prevention, Chinese Center for Disease Control and Prevention, Changping, Beijing, China

75 Shanghai Public Health Clinical Center \& Institutes of Biomedical Sciences, Fudan University, Shanghai, China

76 Division of Clinical Research (DCR), Integrated Research Facility at Fort Detrick (IRF-Frederick), National Institute of Allergy and Infectious Diseases (NIAID), National Institutes of Health (NIH), B-8200 Research Plaza, Fort Detrick, Frederick, MD 21702, USA 